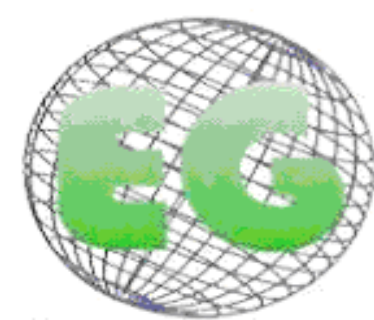

ISSN 1695-6141 $\mathbf{N}^{\circ} 22$
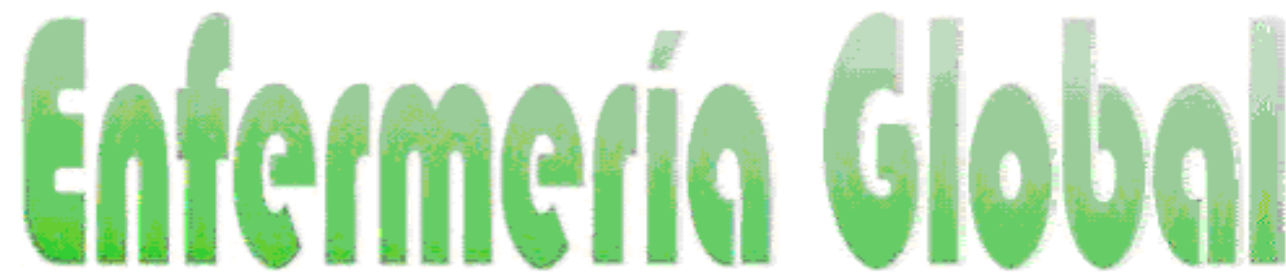

Revista electrónica trimestral de Enfermería

Abril 2011

\title{
EL ENFERMERO FRENTE AL PACIENTE SIN POSIBILIDAD TERAPÉUTICA: DIGNIDAD Y CALIDAD EN EL PROCESO DE LA MUERTE
}

\section{O ENFERMEIRO FRENTE AO PACIENTE FORA DE POSSIBILIDADE TERAPEUTICA: DIGNIDADE E QUALIDADE NO PROCESSO DO MORRER}

${ }^{*}$ De Oliveira Furtado, AM., "*Silva de Souza, SR de O., ${ }^{* *}$ Da Silva Ramos, J., ${ }^{* * * *}$ Ferreira, M de CA.

\begin{abstract}
*Enfermera. Especialista en Nefrología . **Enfermera. Jefa de la Unidad de Cuidados Intensivos del Hospital Pedro Ernesto. ${ }^{* * *}$ Enfermera de la Unidad Semi Intensiva del Hospital Pedro Ernesto. ${ }^{* * * *}$ Enfermero. Especialista en Cuidados Intensivos. Rio de Janeiro. Brasil.
\end{abstract}

Palabras clave: Enfermero; Paciente sin posibilidad terapéutica; Dignidad/calidad en el morir.

Palavras chave: Enfermeiro; Paciente fora de possibilidade terapêutica; Dignidade/qualidade no morrer Keywords: Nurses, Patient outside possibility; Dignity/quality in die.

\section{RESUMEN}

Se trata de una investigación de carácter descriptivo de enfoque cualitativo, basado en la percepción y la preparación del enfermero frente al paciente sin posibilidad terapéutica. Los datos permitieron notar que existe una preocupación de los entrevistados con la preparación para lidiar con la muerte y el morir, hecho que aún posee muchas limitaciones. Pese a ello, el cuidado es la única práctica que no termina frente a las distintas situaciones en las cuales el paciente se encuentra.

\section{RESUMO}

Trata-se de uma pesquisa de caráter descritivo de abordagem qualitativa, tendo como base a percepção e preparo do enfermeiro frente ao paciente fora de possibilidade terapêutica. Os dados permitiram observar que há uma preocupação por parte dos entrevistados com o preparo para lidar com a morte e o morrer, fato este que ainda sofre muitas limitações. Apesar destas o cuidado é a única prática que não cessa diante das diversas situações em que o paciente se encontra. 


\section{ABSTRACT}

This is a descriptive character search with a qualitative approach based on the perception and preparation of the nurse faced with patients for whom there is no therapy possible. The data reveal that there is a concern on the part of interviewees with preparedness to deal with death and dying, an area that still suffers many limitations. Nevertheless, care is the only practice that does not end with the various situations in which patients findthemselves.

\section{INTRODUCCIÓN}

Tradicionalmente, las Unidades de Cuidados Intensivos (UCI) se destinan al tratamiento de los pacientes graves, posibles de recuperación, o con riesgo de muerte, lo que exige que la unidad sea equipada con recursos humanos y materiales. Por lo tanto, las UCI concentran recursos humanos y tecnológicos sofisticados, generalmente no disponibles en otras áreas del hospital, proporcionando, de esta manera, asistencia considerada como una de las más complejas del sistema de salud ${ }^{1}$.

La UCI es un sector que aloja pacientes gravemente enfermos necesitando de cuidados especiales, entre esos cuidados están la preocupación por la calidad de la asistencia. Calidad que está directamente relacionada con la satisfacción o con la superación de las expectativas del cliente; siendo él, el centro de las atenciones de toda la organización. Esa calidad significa implicar a todas las personas, desde la más alta administración hasta el responsable de las tareas más simples ${ }^{2}$.

Pensar en los cuidados intensivos conlleva depararnos con la enfermedad en un alto nivel de complejidad y con el límite entre la vida y la muerte ${ }^{3}$. Un aspecto muy relevante para el equipo intensivista es la paradoja vivida por éste. La UCI es un universo donde se suman la tecnología punta, el conocimiento científico actualizado y la competencia técnica de los profesionales. Así, en la imaginación de cada uno, se instala el poder de las grandes decisiones y del control de la vida y la muerte; no obstante, el paciente, en la dinámica del vivir/morir, deshace esa ilusión de "controlarlo todo". Es allí entonces cuando caemos de la omnipotencia, frustración y de la "incompetencia"; cuando la muerte nos apunta nuestro límite, nuestro término; lo que puede llevarnos a evitar el contacto con el otro, y en consecuencia, con uno mismo.

Aunque enfrentemos situaciones de difícil control, el cuidado permanece, siendo el instrumento/objeto de trabajo del enfermero; más que la cura; por lo tanto, aunque no hayan esperanzas de vida, de cura de una enfermedad, habrá cuidados que realizar. Somos cuidadores y en la UCI vivimos de cerca la cura y el cuidado; cuidado, que "cura", considerando cura como la integración de las distintas partes de la persona que quedaron fragmentadas con la enfermedad. Es la presencia humana lo que hace la profesión imprescindible, esencial y que jamás será substituida por máquinas ${ }^{3}$. Cuidar es un acto individual.

Este estudio tiene el objetivo de analizar la percepción del enfermero frente al paciente sin posibilidad terapéutica en la Unidad de Cuidados Intensivos, averiguar las principales acciones de los enfermeros frente a la muerte en su práctica diaria y discutir los aspectos relevantes en la preparación del enfermero frente al proceso del morir. 


\section{METODOLOGÍA}

Se trata de una investigación de carácter descriptivo, con enfoque cualitativo; el objeto de este estudio está basado en la percepción y la preparación del enfermero frente al paciente sin posibilidad terapéutica. Estudio realizado en un Hospital Universitario del Municipio de Rio de Janeiro.

Los datos fueron recogidos con la aplicación de un instrumento construido a partir de los objetivos propuestos a través de una encuesta semiestructurada con preguntas abiertas y cerradas. Los sujetos sometidos a esta encuesta fueron todos los enfermeros de la Unidad de Cuidados Intensivos de éste mismo hospital.

En este estudio fueron respetados los preceptos éticos de la resolución 196/96 del Consejo Nacional de Salud que trata de las directrices sobre investigaciones que abarcan seres humanos.

El trato de los datos cualitativos fue analizado de forma categorizada, dando énfasis al habla de los sujetos más frecuente y de mayor relevancia para la investigación.

\section{RESULTADOS Y DISCUSIÓN}

La muestra fue compuesta por 18 enfermeros que componen el equipo de funcionarios del sector de Cuidados Intensivos de un hospital universitario durante el horario diurno y nocturno. Fueron agrupados del 1 al 18, precedidos por la letra E. En primer lugar, se describirá el perfil de los participantes en relación a la edad y al tiempo de formación, haciendo una comparación con el momento en que tuvieron preparación para luchar con la muerte.

Tabla 1.- Perfil de los participantes según edad y tiempo de formación en relación al momento en que tuvieron preparación para lidiar con la muerte.

\begin{tabular}{|l|l|l|l|}
\hline Grupo & Edad & Tiempo de formación & Momento en que tuvo preparaci \\
\hline E1 & 43 & 18 años & graduación \\
\hline E2 & 27 & 2 años & graduación \\
\hline E3 & 28 & 4 años & graduación \\
\hline E4 & 37 & 16 años & prácticas \\
\hline E5 & 30 & 6 años & prácticas \\
\hline E6 & 24 & 2 años & no hubo \\
\hline E7 & 30 & 18 meses & no hubo \\
\hline E8 & 29 & 1 año & graduación \\
\hline E9 & 47 & 25 años & no hubo \\
\hline E10 & 25 & 20 meses & en el trabajo \\
\hline E11 & 42 & 16 años & no hubo \\
\hline E12 & 24 & 1 año & no hubo \\
\hline E13 & 47 & 23 años & en el trabajo \\
\hline E14 & 45 & 10 años & posgrado \\
\hline E15 & 45 & 6 años & educación familiar \\
\hline E16 & 46 & 23 años & propio vivir \\
\hline
\end{tabular}


La muerte es un evento que acompaña la vida profesional de todo enfermero, evidenciando que debe haber una preparación para tal situación. De los 18 entrevistados, solo 4 (22.2\%) tuvieron una preparación durante su formación académica; 6 (33.3\%) negaron cualquier preparación; 6 (33.3\%) tuvieron contacto con la situación en el ambiente de trabajo; 1(5.6\%) dijo que fue a través de la educación familiar; y 1(5.6\%) dijo que fue en su propia vida. Eso nos trae un desafío: la preparación profesional, evitando inseguridades y estrés emocional frente a la situación. Fue posible constatar que no existe mucha diferencia entre los enfermeros formados hace mucho tiempo (superior a 10 años), con los recién formados (inferior a 10 años), en relación a la preparación frente a la muerte y el morir. Tal hecho nos muestra que no hubo cambios significativos en la formación de los futuros "enfermeros" frente a esta situación.

Aunque gran parte de los enfermeros no tuvo preparación para luchar con la muerte, mucho de ellos la ven como un proceso natural, con un porcentaje del $61.1 \%(11)$, debido a su preparación que ocurrió en el ambiente de trabajo.

\footnotetext{
Gráfico 1: Indaga sobre cómo el enfermero enfrenta el proceso de la muerte en el ambiente de trabajo
}

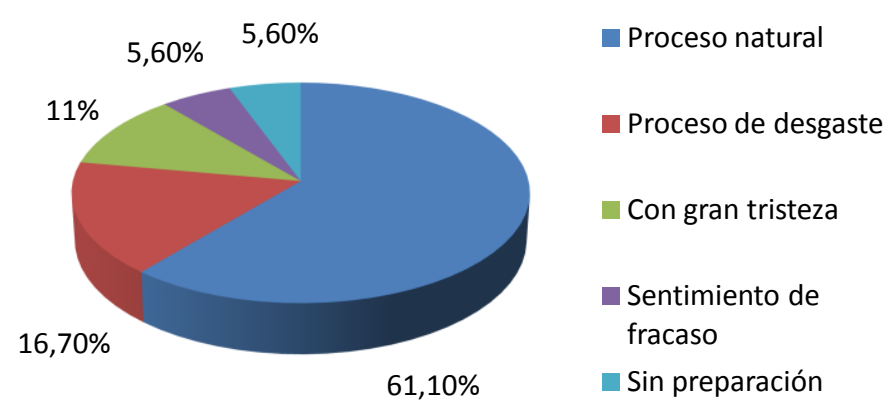

Sin embargo, eso no quiere decir que el enfermero sea un ser "frío", sino que buscó medios adaptativos para enfrentar las más diversas situaciones. Podemos confirmar o confrontar algunas de ellas conforme lo citado por los sujetos:

E12: "Pese a creer en la muerte como un proceso natural, en el fondo se espera que el paciente mejore y vuelva a su casa" /

E13: "Lo veo como un descanso, principalmente cuando hay sufrimiento"/

E16: "Aunque creyéndola natural para pacientes críticos, no acepto la muerte. Es necesario apoyo espiritual y psicológico"/

E10: "Consigo restringirlo a mi trabajo, sin llevarlo para la casa o quedarme conmovida". 
Otros consideran un proceso de desgaste 16.7\%(3), ver Gráfico 1. En el ambiente terapéutico de la UCI se realizan muchos procedimientos diariamente en pacientes con riesgo de muerte o sin posibilidad terapéutica, favoreciendo muchas veces el estrés de los profesionales; sin embargo, el equipo de salud busca proporcionar, a través de su desempeño, un trabajo de calidad $^{5}$.

\section{E5: "Estresante, sin embargo forma parte de nuestro trabajo"/}

E15: "Puede parecer que somos fríos, pero esta situación nos pone frente a dilemas familiares vividos o que iremos a vivir".

El 11\% (2) representa aquellos que consideran la muerte un momento en el que se sienten muy tristes (Gráfico 1).

E6: "No significa tan solo el hecho de ser la muerte de una persona, sino todo el sufrimiento que envuelve a la familia. El contacto con ellos (familia) es peor."

El discurso de nuestra formación es que el cuidado debe ser humanizado, pero el enfermero debe mantenerse lejano emocionalmente de los pacientes asistidos en la unidad de cuidados intensivos. Ante esa realidad, cabe al equipo profesional sensibilizarse para cuidarlo de forma humanizada e individualizada, conduciendo a la familia acompañante, favoreciendo la formación del vínculo que se encuentra fragilizado por este proceso de hospitalización ${ }^{6}$.

El ser humano es singular (único) y el profesional no debe ser visto como un caso aparte; algunos se sienten fracasados ante la muerte en un 5.6\%(1). Aparte del sentimiento de no estar preparado en un 5.6\% (1), lo que nos hace reflexionar sobre la importancia de la atención que debiera ser dada a este tema. Se cree que la preparación profesional puede reflejarse en una asistencia más efectiva para el paciente y con menos estrés para el profesional.

En la pregunta 3 que aborda si el enfermero cree importante la preparación para lidiar con la muerte, $100 \%$ de los enfermeros respondieron que sí.

E1: "Lo creo importante para la salud mental. Debiera ser incluido en la malla curricular"/

E6: "Ayudaría a nuestras emociones"/

E8: “Ayudaría a entender que es una etapa del proceso de vivir”/

E7: "Es importante porque la universidad no nos prepara"/ "para respetar, tratar con dignidad, saber estar con la familia y con uno mismo"/

\section{E14: "Porque atenderíamos con más calidad"}

El enfermero es el eslabón que promueve la comprensión de la enfermedad y el proceso de la muerte, hace que el evento sea más controlable para el paciente y la familia, debiendo ser un defensor de la persona en la etapa terminal y trabajar para garantizar los derechos de esa persona a tener una "muerte tranquila" o a morir con dignidad". En nuestra historia de vida, en nuestra concepción del mundo, de la vida y la muerte. Seguramente, mucho más que un profesional altamente competente 0 eficiente 
técnicamente, el cliente fuera de posibilidad terapéutica necesita de un ser humano que lo conforte, que lo cuide 8,10 .

La Tabla 2 (ver en el anexo 3) muestra uno o más mecanismos de los más frecuentemente utilizados por los enfermeros, mostrando que cada uno posee características singulares para enfrentarse con el proceso de muerte, estando él preparado o no. Pese a las más variadas situaciones adoptadas, el cuidar fue la única práctica que no cesó. El cuidado es un acto individual prestado constantemente por la enfermería.

Tabla 2.- Conductas que suele adoptar frente a la muerte de un paciente

\begin{tabular}{|l|c|c|}
\hline \multicolumn{1}{|c|}{ Actitudes adoptadas } & № & $\%$ \\
\hline Participa del luto & 7 & 38.8 \\
\hline Queda muy conmovido & 3 & 16.7 \\
\hline Consigue esquivar la situación & 14 & 77.8 \\
\hline Lleva la situación de estrés o tristeza hacia afuera del trabajo & 11 & 61.1 \\
\hline Limita el estrés o tristeza al trabajo & 7 & 38.8 \\
\hline
\end{tabular}

\section{CONCLUSIÓN}

Los cuidados de enfermería engloban la provisión de comodidad, manutención de vida, atendimiento de las necesidades físicas y emocionales. La preparación del profesional para lidiar con la muerte y sus procesos significa crear estrategias, lo que, en la mayoría de las veces, va acompañado de estrés emocional y desgaste mental para el equipo de salud, el paciente y el familiar.

Con el estudio fue posible darse cuenta que luchamos, aunque sin preparación adecuada, pero que ofrecemos comodidad y dignidad al paciente sin posibilidad terapéutica y luchamos como profesionales comprometidos con la necesidad del otro. Pero, sobre todo, necesitamos entender que tener la preparación profesional no impide sentirnos angustiados y tristes ante la situación.

Resulta evidente que el cuidado -práctica inherente al enfermero- es el único que no cesa frente a las diversas situaciones en que el paciente se encuentra.

Sería de gran valor para el paciente y los profesionales de enfermería que el proceso de muerte/morir fuese más investigado durante su formación.

\section{REFERENCIAS}

1-Silva MCM,Sousa RMC.Aversão simplificada do therapeutic intervention scoring system e seu valor prognóstico.Rev.Esc.de Enfermagem-USP,2004;38(2):217-24.

2- Knobel E.Terapia Intensiva:Enfermagem.São Paulo:atheneu,2006.

3-Silva LD. Cuidados ao paciente crítico:fundamentos para enfermagem.Rio de Janeiro:Cultura Médica,2.ed.,2003. 
4-Coll'iere MF. Promover a vida.Coimbra-sindicato dos enfermeiros portugueses,2.edição:Lidel,1999.

5- Santos l et al.

Hospitalar:realidade,questões,soluções.São Paulo:Atheneu,vol.2,2005.

6-Chaves EMC, Falcão LM,Fialho AVM,Monteiro ARM,Silva L F.Humanização e tecnologia na unidade de terapia neonatal.Revista Nursing,2007;10(113):467-470.

7-Smeltezer SC, Bare BG.BRUNNER \& SUDDARTH. Tratado de Enfermagem Médicocirúrgica. 9a ed. Rio de Janeiro: Guanabara Koogan, 2002.

8-Arruda EM, Gonçalves LH.A enfermagem e a arte do cuidar. Florianópolis: Univ.de Santa Catarina,1999.

9-Rider JE. Enfermagem conteporânea:desafios,questões e tendências,5.ed, Porto alegre:artmed,1998.

10-Prado C, Santos SN. Reflexões sobre o processo de humanização da equipe de enfermagem. Nursing, 2008;11(119):189-194. 


\section{APÉNDICE I}

Edad-

Tiempo de formado

1-¿En qué momento tuvo usted preparación para lidiar con la muerte?

()en la graduación/carrera ()posgrado ()cursos ( )otros--------------

2-¿Cómo usted enfrenta el proceso del morir en su ambiente de trabajo?

3-¿Considera usted importante la preparación para lidiar con la muerte?
( ) sí
( ) no

4-Señale la (las) conducta(as) que usted suele tener con la muerte de un paciente:

( ) apatía

( ) se siente triste y se aleja de aquella realidad

( ) participa del luto con la familia

( ) se siente muy estresado(a) con la situación

( ) sufre una gran conmoción (emocional)

( ) consigue lidiar bien con la situación (profesionalmente)

( ) lleva la situación de estrés y/o tristeza fuera del ambiente de trabajo

( ) limita esa situación(estrés y/o tristeza) solo al ambiente de trabajo 